\title{
A variation in the course of sciatic nerve in relation to the piriformis muscle: A case report
}

\author{
Chandrasiri DACT ${ }^{1} *$, Patabandige $\mathrm{DJ}^{1}$, Ekanayaka EADA ${ }^{1}$, Dissanayaka $\mathrm{JK}^{1}$ \\ ${ }^{\prime}$ Department of Anatomy, Faculty of Medicine, University of Peradeniya. \\ *dineshdr17@gmail.com
}

Non discogenic sciatica is a disease, where the sciatic nerve $(\mathrm{SN})$ or its branches, the Common Peroneal Nerve $(\mathrm{CPN})$ and the Tibial Nerve (TN) compressing against the piriformis muscle (PM) because of its anatomical relation to the PM. Understanding about these variations is important in early diagnosis of Piriformis syndrome (PS) in clinical medicine. Twenty seven cadavers (53 lower limbs) used for routine undergraduate dissections at the Department of Anatomy, Faculty of Medicine, University of Peradeniya, Sri Lanka, were studied and the course of the $\mathrm{SN}$ as it travels in relation to the PM was observed and classified according to the Beaton's and Anson's classification. Of the 53 lower limb specimens observed, there was 01 $(1.88 \%)$ case, where the $\mathrm{CPN}$ was emerging through the piriformis muscle to begin with, while the TN passes below the PM (Type II of Beaton's and Anson's classification). It was a right limb, and the left lower limb was having normal anatomy (Type I). Other specimens $(98.22 \%)$ were showing the normal anatomical variation (Type I). The Type III and type IV of aforementioned classification was not observed. The normal course of SN is that it emerges below the PM (Type I). In the present study a variation of SN which can lead to PS with type II variation was observed. Therefore, knowledge on anatomical variations of the $\mathrm{SN}$ is important, in diagnosing the syndrome. Having high clinical suspicion will make the treatments much easier, and it will be helpful for the radiologists as well. 